\title{
MOBILITY MANAGEMENT FOR UNTETHERED IMMERSIVE COMMUNICATIONS
}

\author{
Mehran Dowlatshahi, Farzad Safaei \\ Smart Internet Technology CRC, University of Wollongong
}

\begin{abstract}
In this paper we propose a system design for delivery of immersive communications to mobile wireless devices based on a distributed proxy model. It is demonstrated that this architecture addresses key technical challenges for the delivery of these services, that is, constraints on link capacity and power consumption in mobile devices. However, additional complexity is introduced with respect to mobility management. The paper proposes three possible methods for updating proxy assignments in response to mobility and compares the performance of these methods.
\end{abstract}

\section{INTRODUCTION}

There has been a significant increase in popularity of Networked Virtual Environments (NVE) in recent years. For example, reliable estimates indicate that by 2009 more than 230 million people will be playing multiplayer network games and, in particular, mobile games show significant growth [1].

Natural human communication within an NVE requires creating a suitable multimedia scene (voice, video, gestures and haptics) for each participant to mimic the real world sensory information of being in the presence of a group or a crowd. The audio scene, for example, must include the voices of all avatars in the participant's hearing range, spatially placed at a suitable distance based on the participant's perspective. Unlike the current person-to-person communication services, which are characterized by more or less static point-to-point traffic flows, immersive communication involves a myriad of point-to-multipoint flows with highly dynamic changes in their connectivity arrangements. For example, the voice of each participant has to be included in the audio scene of everyone within the audible range of this voice. Likewise, other multimedia content (visual, gesture and haptics information) sourced from a given participant should reach everyone who is 'interested' in this information. Conceptually, one might view several parallel multicast flows from the source to others within the area of interest. This 'area of interest' may differ for different types of media. Voice, for example, could propagate through walls while visual information does not. As avatars move within the virtual environment, these multicast trees must undergo change. The immersive communications, therefore, is characterized by a large number of multicast flows that are subject to rapid reconfiguration.

Wireless access to multimedia immersive communications presents additional challenges. It would be desirable to relieve wireless clients from excessive low level flow management functions (such as continuous changes in required media streams in response to mobility in the virtual environment). The mobility of wireless devices across networks will create new challenges for mobility management and require new functions in addition to layer 2 and layer 3 handovers to control latency.

Rest of the paper is organized as follows. Section 2 describes our proposed system architecture. Section 3 provides a possible solution for managing physical mobility and simulation results on its effectiveness. Section 4 presents the concluding remarks

\section{SYSTEM ARCHITECTURE}

A conceptually simple model for wireless immersive communications is to use a peer-to-peer model for transmission of multimedia content between the participants. To use a concrete example, let us consider immersive voice communications for the following discussions. In a peer-to-peer model, each client captures the voice of its user and must identify the subset of participants who would be interested in this voice stream. The voice stream is then multicast to this subset. Alternatively, multiple unicast flows may be used if the underlying network cannot support multicast.

While simple, this model has some drawbacks. First, the downstream wireless link is a shared media and would limit the number of flows that can be received by each participant. Second, the clients have to participate in rapid reconfiguration of multicast flows in response to application dynamics (such as movement of avatars in the virtual world), which is a processing burden on wireless devices.

To overcome these difficulties, we propose to use a set of distributed servers - referred to as proxies - to aid in the delivery of multimedia streams to clients. Each proxy is responsible for a group of clients and, in essence, performs the necessary functions of these peers on their behalf.

Figure 1 shows this architecture for a small NVE. Every wireless client is connected to a proxy server. To improve latency, it would be best to connect the client to its closest (in terms of network delay) proxy.

On the upstream side, the client will send its voice packets to its proxy. It is the responsibility of the proxy to forward/multicast this voice stream to other proxies who might need this information for the creation of their clients' audio scenes. This is shown in Figure 1. Proxy P1 receives voice packets from one of its clients (avatar 1). P1 will determine the audible range of this signal by analyzing its loudness and the characteristics of the environment (for example presence of sound barriers such as walls). The audible range is shown as a closed area in this Figure which includes several avatars, namely avatars $2-6$. P1 will then determine the proxies for avatars 2-6 which happen to be P3, P4 and P5. Avatar 1 's voice packets are then multicast to $\mathrm{P} 3, \mathrm{P} 4$ and $\mathrm{P} 5$ with $\mathrm{P} 1$ as the root of the overlay multicast tree. Similarly, the proxies

This work is sponsored by Smart Internet Technology Co-operative Research Center. (SIT-CRC) 
associated with all other (talking) avatars will create overlay multicasts for the purpose of communicating their client's voice. On the downstream side, the proxy should send the audio streams of relevance to its connected clients.

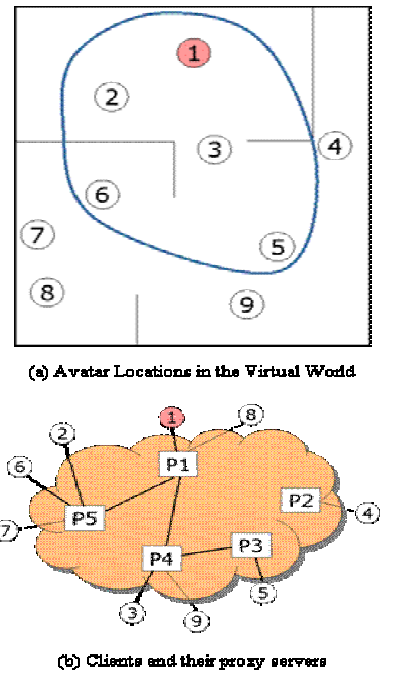

Figure 1. Distributed Proxy Architecture for Immersive Communications

\subsection{Coping with mobility in the virtual world}

In the distributed proxy architecture, the wireless devices need not participate in formation and reconfiguration of multicast trees. Instead, each proxy will become the root of multicast for all of the streams sourced by its attached clients and will have to join all those multicast trees associated with streams needed by its clients. Apart from the fact that this will make the task of wireless devices much simpler, using proxies as opposed to peers for formation and reconfiguration of multicasts has another important advantage: the reconfiguration of multicast trees due to movements in the virtual world happens less often.

To illustrate this point consider Figure 1 once again. The movement of avatars will change the composition of crowds and the proximity of avatars to each other within a crowd. Consequently, the list of avatars in one's audible range will change due to movement of both the speaker and the listeners. This, in turn, may lead to a new multicast tree if any of the proxy leafs are different. Given that proxies are participating in multicast trees on behalf of all their attached clients, this change happens less often than a peer-to-peer model. For example, in Figure 1 if avatar 2 moves out of the audible range of avatar 1, the multicast tree from P1 will not change because there is still another avatar (6) connected to the same proxy that needs avatar 1's voice stream. In recent years many techniques for construction of overlay multicast trees and networks have been proposed [2, 3, 4]. In [5] we have proposed an algorithm for construction of overlay multicast trees that is scalable to a large number of highly dynamic trees and will allow rapid reconfiguration on the time scales which are consistent with movements within a virtual environment.

\section{MOBILITY MANAGEMENT}

In the previous section we demonstrated that using a distributed proxy architecture can significantly improve scalability and robustness of immersive communication services for wireless nodes. The use of distributed proxies, however, creates an additional complexity - mobility management. This is particularly pertinent if the underlying network topology is hierarchical, which is of course very common.

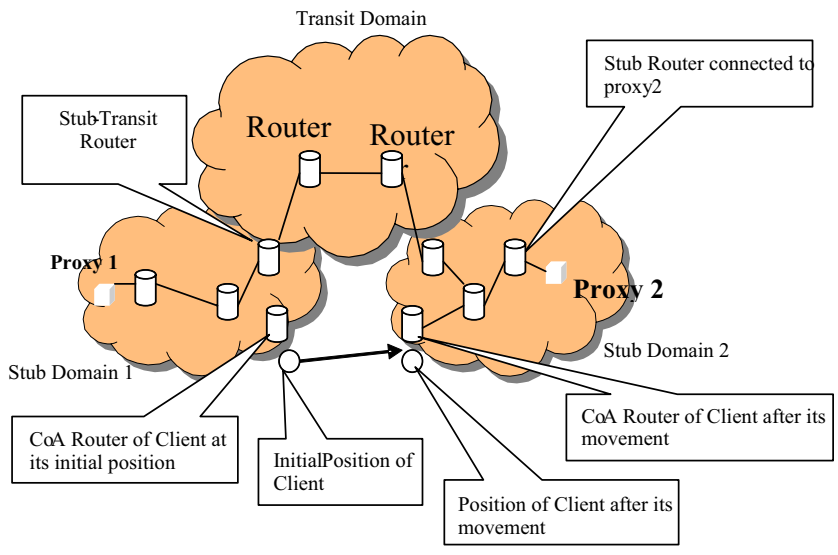

Figure 2. Movement of wireless node from one stub domain to another

To illustrate this point, consider the network of Figure 2 where a portion of a hierarchical infrastructure comprised of two stub domains interconnected using a transit domain is shown. A wireless client is initially connected to stub domain 1 through one of the routers associated with this domain as its care of address $(\mathrm{CoA})$ router [6]. Let us assume that the client is mobile. Figure 2 shows a case when the mobile node has moved outside the range of its stub domain and connected to a new CoA router. Given timely handovers using layer 2 and layer 3 , it should be possible for the wireless node to continue its multimedia communication session. However, the location of its proxy may no longer be suitable. For example, the proxy may be connected to the old stub domain and the communication between the new CoA router and this proxy may have to go through one or more transit domains and experience significant increase in delay (although in terms of geographical distance the change may not be as significant). In this case, it may be important to reduce the latency by assigning the client to a closer (in terms of network delay) proxy such as the proxy in stub domain 2 . The key issue is that a change of stub domain could happen as a result of moderate movement but may lead to significant increase in network delay from the wireless device to its proxy.

In our simulation experiments we use a Transit-Stub topology [7] to simulate a two-layer hierarchical topology. The network consists of six transit domains, each with an average of 10 routers. Each transit router is connected to an average of 3 stub domains, and each stub domain consists of 8 routers. Routers at any of the transit or stub domains have an average of 3 physical links to the network. The network is assumed to represent a 5000 by $5000 \mathrm{~km}$ geographical area. Note that we are not implying that different domains are owned by different network providers. A hierarchical infrastructure is common for large scale carriers that operate across a vast geographical area (such as USA or Australia)

In Figure 3 the percentage of moves that lead to a change of stub domain is shown for various ranges of movement from 1 to $8 \mathrm{Km}$. As can be seen, the chances of changing stub domain would 
increase as we move further from pervious location. However, compared to the overall size of the network, relatively small moves could lead to significant probability of stub domain change.

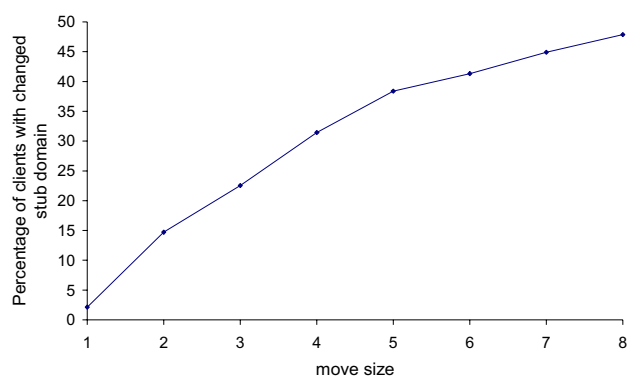

Figure 3. Percentage of stub domain changes versus move size

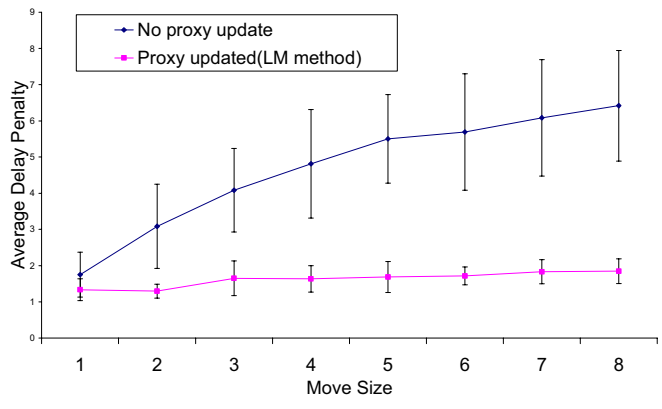

Figure 4. Average delay penalty increase versus move size (in Kilometers)

Figure 3 shows the impact of movement size on the average Delay Penalty ratio between the wireless node and its assigned proxy. The Delay Penalty ratio is defined as the ratio of network delay from the wireless node to its previous proxy over this delay to the closest proxy. As shown in the Figure, without a proxy update mechanism, the latency perceived by the immersive communication service can significantly increase. Since all immersive communication flows to/from the wireless node are sent/received through the proxy, the increased delay penalty also implies wastage of network resources by using a longer than necessary path. For comparison, the delay penalty after a proxy update using the Landmark method (to be described later) is also included.

Summarizing the above observations, there are three possible cases: A) Range of movement of the wireless node is small and Layer 2 handover mechanisms are sufficient to maintain connectivity to the CoA router. In this case, there is no change in the CoA router and no proxy update would be needed; B) The wireless node moves to such an extent that the CoA router changes and a layer 3 handover is triggered. However, the new CoA router is in the same stub domain as the previous CoA router. If there is only one proxy within this stub domain, a proxy update is unlikely to be required. However, if the stub domain covers a vast geographical area and contains multiple proxies (in other words, the network topology is more or less flat in this region), an update may still improve the service delay; C) As in case B above, but the new CoA router resides in a different stub domain. In this case, it is highly probable that a proxy update would be beneficial.

The following observations are relevant to determine the most suitable proxy when an update is required: 1) There is only a very loose relationship between geographical and network proximity.
This is particularly true for a hierarchical network topology as shown in Figure 2 where small changes in geographical proximity may translate to large variations in network delay. Nevertheless, it is quite likely that the optimal proxy (in terms of network latency) is not too far away. 2) We do not wish to burden the wireless node to carry out an exhaustive search for finding the optimal proxy (for example conducting a statistically reliable set of ping time measurements to all proxies after each move). 3) The wireless link (and in particular its MAC layer) could add significant jitter to delay measurements carried out by the wireless devices themselves.

Both observations 2 and 3 above suggest that we need to develop some form of support by the fixed infrastructure for the proxy update mechanism that requires minimal functions from the client.

\subsection{Proxy Update Mechanism}

In this article we propose a mechanism to identify the closest proxy by providing a Proxy Location Register (PLR) facility and a set of known landmarks [8]. The steps in identifying the nearest proxy is summarised below:

Client notices a change in its $\mathrm{CoA}$ and therefore conducts a measurement of its round trip time (RTT) from the landmarks. The client sends its new care of address and the results of its delay measurements from landmarks (and possibly its derived network coordinates in the Coordinate method to be described later) to the PLR. If the PLR already knows the closest proxy to this CoA router, it informs the client of the new proxy and the procedure ends. Otherwise, the PLR determines the closest proxy by the following steps: PLR creates a set of what it considers to be the closest proxies to the CoA router as potential candidates. In this article, we compare three different methods for creating this candidate set and present comparative results on the effectiveness of these. The PLR sends a request message to each of the proxies in the candidate set to measure their RTT from the new CoA router. These measurements are then returned by the candidates to the PLR and may be cached for future use. The PLR determines the closest proxy for the client by selecting the candidate with minimum delay from the CoA router and informs the new proxy and its newly associated client about the update. Note that the above procedure can also be used at the time when a new client joins the immersive communication service and repeated thereafter in response to mobility.

The key step in the above procedure is producing the candidate set of closest proxies based on the client's CoA and its measured delays from the landmarks. Here, we compare three possible methods for this purpose:

1) Coordinate Method: This method is based on modeling the network by a multidimensional geometric space where measured delay between any two nodes is assumed to be proportional to the distance between those two in the geometrical space [9]. The Proxy Location Register in this case has the coordinates of all proxies. Each host (whether proxy or client) based on its RTT measurement from landmarks and coordinates of landmarks finds the optimal coordinates for itself in this space such that the distances in the same space match the measured delays from landmarks as closely as possible. Due to approximation, it is usually not possible to correctly determine topologically closest proxy to a client in the network. Hence, the PLR will choose a fixed number of closest proxies in the geometrical space as the 
candidate set for further measurement of their delay form the CoA router.

2) Landmark Closeness Order: The second method is based on comparing the closeness order of proxies and the client to a set of well known Landmarks [8]. The PLR will then choose the candidate proxies by selecting those proxies with minimum distance in terms of their landmark closeness order. The set of determined proxies is then likely to include the closest proxy to the client. Landmark proximity order is most effective when RTT measurements from landmarks are rather accurate. For wireless hosts, the inaccuracy in RTT measurements may affect the proximity order of landmarks. We have therefore have devised a method for derivation of distances between any two hosts when proximity orders are not accurate.

3) Geographical Position: The third method is based on knowing exact geographical position of the client and proxies. In this method the candidate set is comprised of a number of geographically closest proxies to the client, which is expected to include the topologically closest proxy as well. This method can only be used if the client knows its geographical position, for example using GPS or by receiving its approximate geographical location from its $\mathrm{CoA}$ router. The geographical locations of proxies are also assumed to be known.

Figure 5 shows the delay penalty ratio after the proxy update is completed compared to the optimal proxy (if it could be found). Recall that in all three methods, the candidate proxies are requested to measure their RTT from the CoA router. The proxy having the minimum delay is then assigned to the client. It is possible that the optimal proxy is not within this candidate set. In order to increase the probability of finding the closest proxy, the number of proxies in the candidate set has been increased from 10 to 50 on the horizontal access (i.e. from $5.5 \%$ to $27.7 \%$ of all proxies in the simulated network model). Clearly, having a large number of proxies in the candidate set increases the accuracy but also raises the computation and bandwidth overhead and delay associated with proxy handover.

As shown in Figure 5, by increasing the size of the candidate set the delay penalty ratio decreases. In other words, the likelihood of finding a better proxy increases. The geographical position method can almost always find closest proxy for a candidate set size of 20 or more ( $11 \%$ of all proxies). The Landmark Closeness Order method and Coordinate method performance are inferior but for sufficiently large set sizes same average delay penalty can be achieved.

\subsection{Updating Multicast Trees After a Proxy Update}

Ideally, the proxy update in response to mobility should be seamless and without any disruption to the service. The main purpose of update is to improve delay performance and cost of delivery. It is therefore important to reconfigure multicast trees affected by this update as quickly as possible but without disruption. To this end, it is proposed here to have another facility referred to as Client Proxy Association (CPA) server. (This server may be running on the same hardware as the Proxy Location Register if appropriate.) The CPA server maintains a list of every client/avatar and their assigned proxies. After Proxy Location Register completes proxy update of a mobile client, the PLR will update the client's entry in the CPA server with the new proxy. All proxies are required to consult $\mathrm{CPA}$ in constructing or reconfiguring their multicast trees after new client allocations as well as on regular time intervals.

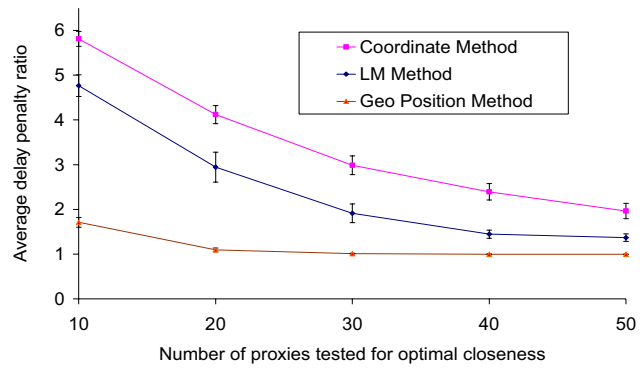

Figure 5. Average Delay Penalty with respect to size of the candidate set $(20 \%$ wireless delay variation)

Old proxy of a moved client should forward required flows of the moved client to the new proxy of the client while necessary. This policy assures uninterrupted forwarding of the media streams to the moving clients.

\section{CONCLUSIONS}

Immersive communications is likely to form the basis of many future services for collaborative work, education and play. Access to these services with mobile devices will be of significant commercial interest. It is important to design a system architecture that can cater for both wired and wireless access. In this article, we have proposed an architecture that can achieve this goal. The distributed proxy model is suitable for both fixed and mobile clients and reduces the required functionality performed by the wireless nodes. We have also proposed possible methods for updating proxies in response to mobility. The role of the wireless device in managing mobility is still minimal and therefore the impact on the wireless link usage and power consumption remains negligible.

\section{REFERENCES}

[1] DFC Intelligence, "The Online Game Market 2004", August 2004, California, USA.

[2] S. Banerjee, B. Bhattacharjee and C. Kommareddy. "Scalable Application Layer Multicast." In Proc. of ACM SIGCOM, 2002.

[3] J. Liebeherr, M. Nahas, and W. Si, "Application-Layer Multicasting With Delaunay Triangulation Overlays," IEEE JSAC, Volume 20, Number 8, October 2002

[4] Eli Brosh, Yuval Shavitt, "Approximation and Heuristic Algorithms for Minimum Delay Application-Layer Multicast Trees", in Proc. Of IEEE Infocom, 2004

[5] Dowlatshahi, M., Safaei, F., "A Recursive Overlay Multicast Algorithm for Distribution of Audio Streams in Networked Games", proceedings of the IEEE International Conference on Networks (ICON), November 16th-19th, 2004, Singapore.

[6] C.E. Perkins 'Mobile IP' IEEE Communications Magazine - May 2002

[7] K. Calvert, E. Zegura, and S. Bhattacharjee, "How to Model an Internetwork," in Proc. IEEE Infocom, 1996.

[8] S. Ratnasamy, M. Handley, R. Karp, and S. Shenker,'Topologicallyaware overlay construction and server selection'In INFOCOM'02, New York, 2002. IEEE.

[9] T. S. Eugene $\mathrm{Ng}$ and Hui Zhang, "Predicting Internet Network Distance with Coordinates-based Approaches", Infocom 2002, P170179. 